\title{
L'intérêt de la modélisation comme aide à la rédaction en anglais pour les scientifiques français
}

\author{
Elizabeth Crosnier
}

\section{OpenEdition}

\section{Journals}

Édition électronique

URL : http://journals.openedition.org/asp/3439

DOI : 10.4000/asp.3439

ISSN : 2108-6354

Éditeur

Groupe d'étude et de recherche en anglais de spécialité

Édition imprimée

Date de publication : 1 décembre 1996

Pagination : 89-103

ISSN : 1246-8185

\section{Référence électronique}

Elizabeth Crosnier, «L'intérêt de la modélisation comme aide à la rédaction en anglais pour les scientifiques français », ASp [En ligne], 11-14 | 1996, mis en ligne le 29 avril 2013, consulté le 19 avril 2019. URL : http://journals.openedition.org/asp/3439; DOI : 10.4000/asp.3439

Ce document a été généré automatiquement le 19 avril 2019

Tous droits réservés 


\title{
L'intérêt de la modélisation comme aide à la rédaction en anglais pour les scientifiques français
}

\author{
Elizabeth Crosnier
}

\section{Introduction}

1 Avant d'aborder la question de la modélisation et de ses applications pédagogiques qui sont actuellement au centre de nos préoccupations, nous tenons à rappeler en introduction quelques éléments d'informations. Les anglicistes qui enseignent dans des milieux scientifiques connaissent déjà le public et leurs besoins, mais, en revanche, les "nouvelles recrues » découvriront peut-être des situations peu familières et parfois déroutantes.

2 De nos jours, la rédaction d'articles en anglais fait partie des activités courantes des scientifiques. La parution dans les actes de congrès ou les périodiques hautement spécialisés dépendant souvent de Societies ou associations représente l'objectif premier des chercheurs et ingénieurs qui souhaitent informer leurs pairs de l'évolution de leurs travaux. Cette course à l'information s'accélère régulièrement et l'inflation en matière de publication est galopante. Une telle situation, dont il ne semble pas nécessaire de rappeler l'historique dans ces pages, ne fait qu'amplifier les problèmes qui se posent aux Français lorsqu'ils sont contraints d'utiliser la langue anglaise comme outil de communication. Actuellement, face à ce passage obligé, ils n'ont plus le choix et ils doivent suivre ce processus face à l'internationalisation de la science d'une part, et l'hégémonie de la langue anglaise de l'autre. En outre, la confrontation inéluctable avec les réviseurs anglophones des comités de lecture représente un obstacle supplémentaire. Dans cette recherche, nous n'avons envisagé que l'échange entre scientifiques français et des lecteurs anglophones ; l'idée de communication scientifique en milieu international a été volontairement exclue car elle nécessiterait de nouvelles investigations. 
3 Les enseignants d'anglais en milieu scientifique français réalisent l'importance de l'enjeu et se voient dotés d'une mission relativement nouvelle : offrir aux auteurs, futurs ou confirmés, les outils indispensables en mesure de les aider à affronter les difficultés de la rédaction de l'article scientifique dans une langue autre que leur langue maternelle. L'acceptation par le comité de lecture dépend notamment de deux facteurs qui nous intéressent du point de vue langagier (les critères d'acceptation des articles, multiples et complexes, n'ont pas leur place ici) : développer une maîtrise de la langue suffisante pour i) transmettre le message dans son intégralité et ses subtilités, ii) offrir les meilleures conditions de lisibilité.

4 Il ne paraît plus nécessaire de rappeler la nécessité de dépasser le stade purement linguistique. De ce point de vue, à savoir de par sa connaissance des deux communautés de langue et de leur environnement socio-culturel, le linguiste doit pouvoir ainsi aborder la langue dans sa globalité et par conséquent inciter les scientifiques à porter leur regard sur des phénomènes qu'ils ont négligés jusqu'alors. De cette manière, il s'intéresse à un genre d'écrit spécifique en s'interrogeant sur les paramètres qui interviennent dans cette communication :

- profil professionnel et socio-culturel des auteurs français ;

- profil professionnel et socio-culturel des lecteurs anglophones, récepteurs de messages ;

- conditions d'utilisation de la langue et de transmission des informations.

Suite à cette démarche, et pour résumer l'essentiel, il est possible de savoir qui dit quoi, à qui, pourquoi, où, comment, et de noter que les différences majeures portent sur le profil socio-culturel des interlocuteurs.

C'est à partir de cette étape d'intégration de l'article scientifique comme genre qu'il est possible de rechercher, dans un corpus d'écrits en français et en anglais, les spécificités langagières, les marques de conventions et de règles de bon usage en vigueur dans cette communauté socio-professionnelle. L'étape suivante consiste à effectuer l'étude contrastive des résultats pour chaque corpus afin de mettre en lumière les éventuelles similitudes et divergences. En prenant comme référence les écrits anglais, nous émettons l'hypothèse que les disparités relevées dans les écrits français risquent d'être à l'origine des difficultés de leurs auteurs. Si on compare le profil des deux groupes d'interlocuteurs, on se rend compte que les lieux de divergence se situent au niveau culturel, individuel et collectif, chacun portant son propre bagage auquel s'ajoutent les marques de la culture institutionnelle. En conséquence, nous portons notre attention, non seulement sur l'expression du contenu scientifique en anglais et en français de l'article, mais encore sur le transfert de messages qui s'inscrit dans un environnement culturel spécifique.

7 Ce cheminement nous mène maintenant à étudier une communauté liée par des objectifs communs, qui inclut cependant la confrontation d'individus dont la langue et la culture sont extrêmement variables. Nous posons alors comme essentiel le fait que le terrain de compréhension et de connivence ne peut exister que si ces faits sont d'abord explicités puis clairement posés. Ensuite, puisqu'il est souhaitable de combler un fossé susceptible de pénaliser les auteurs français en situation de publiants, ce gap que mentionne Cooke (1993 : 463), où notamment la notoriété est en jeu, les linguistes doivent s'efforcer de développer, en premier lieu, la prise de conscience de certains phénomènes tels que ces conventions en usage dans une communauté précise, de la nature des variations au niveau socio-culturel, individuel et collectif, et enfin des fonctionnements langagiers 
différents pour traduire des notions considérées comme universelles, aspect qui a été souvent nié lorsqu'on aborde le monde scientifique.

\section{Notion de modèle et méthodologie}

Après avoir posé le problème des scientifiques français en situation de rédacteurs en langue anglaise et soumis au verdict des réviseurs anglophones, puis considéré le genre spécifique du message contenu dans l'article scientifique comme dicté par des règles inhérentes à la communauté, et enfin reconnu l'existence de lieux de consensus et de divergence, nous pouvons présenter la notion de modèle et la méthodologie que nous avons tenté d'élaborer à partir de documents authentiques.

9 Cette recherche a été largement motivée par les remarques de réviseurs et de linguistes anglophones, formateurs pour étudiants scientifiques étrangers, mentionnant l'absence de cohérence et l'inaptitude à se conformer aux règles de la rhétorique anglo-saxonne. Nous avons également suivi, et transposé pour l'anglais langue seconde, la démarche d'une équipe d'enseignants-chercheurs en français langue étrangère (FLE) du CLA de Besançon qui se consacre au français de spécialité et aux relations entre la langue scientifique et la culture, dont voici quelques propos :

Toute cohérence s'élabore en fonction de son public et s'y adapte. Écrire, c'est aussi communiquer. Une double difficulté se présente alors au scientifique étranger lorsqu'il doit rédiger un texte :

- d'abord proposer une schématisation en français à une représentation mentale qu'il a construite.

- puis vérifier que cette schématisation est perçue par le destinataire français ou

francophone préalablement défini. (1990: 146)

10 Cette orientation nous a semblé offrir une voie intéressante à exploiter pour l'enseignement de l'anglais de spécialité dans un contexte scientifique français.

11 La modélisation peut être définie comme une opération qui consiste à décrire un processus réel au moyen d'un schéma, ou pattern, simplifié dans le but de l'élucider et de construire un modèle se rapprochant le plus possible de ce processus de départ. Le modèle est validé après avoir vérifié son efficacité. L'objectif de cette opération est de l'utiliser dans le but de réaliser de nouveaux processus.

La notion de modèle issue de la théorie du genre et son application au texte scientifique anglais ont été lancées par quelques linguistes anglophones qui ont effectué l'analyse de discours spécialisés à partir de différentes sections tels que les abstracts, les introductions, les discussions, les résultats, et notamment à J. Swales qui, en précurseur, a fortement marqué la tendance. Sans vouloir dresser l'historique, il semble utile de rappeler les grandes orientations de ce mouvement qui intéresse actuellement les anglicistes dans le cadre d'applications pédagogiques en anglais de spécialité. Quelques ouvrages sont mentionnés à la fin de cet article à titre d'information.

Swales (1981:141) a noté un ordre établi des séquences qui composent l'introduction et a défini un modèle d'organisation. Il l'a ainsi décomposée en moves, chaque move, ou séquence, correspondant à une fonction :

- Move 1 : Establishing a Territory, lorsque l'auteur fait référence à l'existant ;

- Move 2 : Establishing a Niche, phase de critique qui met en avant la nécessité de pallier des lacunes, argumentation, avec des étapes variables selon les auteurs ;

- Move 3: Occupying the Niche, où l'auteur propose ses solutions. 

certaines variantes en fonction des écrits analysés; les moves de Swales ont parfois été remplacés par des cycles. Il faut toutefois préciser que les tendances mettent plutôt en avant des aspects soit fonctionnels, soit linguistiques ou bien encore sociologiques. Dans notre cas et vu notre démarche, ces derniers sont obligatoirement indissociables. En partant des faits langagiers, nous avons ainsi opté pour une approche globale des messages. Par conséquent, hormis ces modèles de structures récurrentes, ceci permet d'ouvrir des pistes d'investigations sur des points généralement occultés tels que les diverses fonctions et stratégies mises en œuvre par les auteurs, la part d'interaction et la finalité sociale qui font de l'article scientifique un acte de communication dépassant largement le simple contenu scientifique.

Notre étude a porté sur des parties d'articles qui peuvent être classées, elles-mêmes, dans un sous-genre puisqu'elles ont leur propre système d'organisation. Il est important de rappeler que l'article est divisé en sections selon une forme canonique en vigueur dans chaque discipline. Nous avons sélectionné des sujets précis appartenant à trois disciplines : l'informatique, les mathématiques et la mécanique. Ce travail a été effectué dans le but de mettre l'accent, en premier lieu, sur les raisons pour lesquelles les scientifiques français éprouvent tant de difficultés à rédiger en anglais. Ensuite, nous avons cru nécessaire de cerner l'origine et la nature des obstacles, convaincue qu'il fallait dépasser le stade de la langue, puisqu'il a été montré que l'absence d'erreur de vocabulaire et de grammaire n'entraîne pas systématiquement la bonne réception du message. Nous avons posé le problème de l'acceptabilité de la langue, du niveau de compréhension et de confort, dans une optique pédagogique.

L'analyse d'abstracts et d'introductions en anglais révèle l'existence de schémas récurrents, de patterns, grâce à un système de repérage de formes langagières traduisant des fonctions, à la fois professionnelles où nous intégrons l'expression propre aux activités scientifiques, et sociales qui traduisent le désir de communication avec l'extérieur et l'interaction entre l'auteur et le lecteur. Ces indices de surface, répertoriés au moyen du programme informatisé d'analyse de textes ALCESTE de Max Reinert (Analyse des léxèmes cooccurrents dans les énoncés simples d'un texte) du CNRS de Toulouse, et notamment les régularités interprétées grâce à un concordancier, permettent de découvrir des systèmes d'organisation des idées, les stratégies de communication, l'art de convaincre et la perception qu'ont les Anglo-Saxons de notions de temps et d'espace, de cohérence ou unité interne, selon la définition donnée dans notre thèse (1995 : 275), de rhétorique. Cette approche notionnelle a elle aussi été dictée suite au repérage de termes et de leurs associations. Notre méthodologie conduit alors à la construction de modèles. En revanche, des textes équivalents produits par des scientifiques français mettent en lumière une plus grande variété de schémas et des divergences au niveau de l'expression de la perception du monde, a priori source de difficultés, comme le montre l'analyse contrastive. Nous exposons ici les grandes lignes de notre démarche ainsi que quelques-uns de nos résultats qui seront validés par le biais d'une première expérimentation. De cette façon, nous essayons de montrer que la modélisation peut apporter une aide non négligeable pour l'enseignement de l'anglais de spécialité à un public de Français. 


\section{Étude contrastive, application aux abstracts d'informatique}

17 Après avoir tenté de définir la modélisation et exposé notre méthodologie, nous présentons brièvement une partie de l'expérimentation avec les abstracts d'informatique qui offrent des résultats particulièrement probants. L'abstract fut découpé en trois composantes : le titre, la première phrase et le texte intégral. Chacune d'elles fut analysée selon la méthode suivante :

1. Analyse informatisée d'un corpus de textes;

2. Repérage et répertoire des formes visibles: expressions et mots récurrents, ordre et associations des mots, expressions composées;

3. Interprétation: rapports entre le visible et l'implicite, la langue et les fonctions professionnelles et sociales, notions, qualité de l'information

4. Construction de modèles ;

5. Observation des résultats et comparaison entre l'anglais et le français;

6. Validation des résultats.

18 C'est ainsi que les titres ont, d'emblée, révélé le rôle joué par des fonctions instrumentales et sociales, mis en lumière la manière de percevoir le temps et apporté des éléments sur la qualité de l'information. La première phrase a mis l'accent sur la qualité du thème, la manière de l'installer et de le traiter, ainsi que sur la notion de cohérence thématique avec le titre, puis fait apparaître la relation de l'auteur avec le monde animé. Pour terminer, le texte dans sa globalité met en avant les différentes fonctions professionnelles et sociales, montre la cohérence de l'ensemble, la perception de l'espace-temps, et enfin tout le schéma d'organisation.

19 Nous avons ainsi relevé les notions de cohérence dans la manière d'organiser la pensée et d'espace-temps à travers l'ordonnancement des idées dans l'espace de l'écrit. Les fonctions liées au contenu scientifique combinées aux expressions de la relation qu'établit l'auteur avec ses lecteurs, les marques de connivence, les intentions qu'il traduit lorsqu'il évalue son travail et les stratégies qu'il développe afin de convaincre du bien-fondé, tout en ménageant des garde-fous, ce que les sociologues et linguistes appellent hedging (protection pour parer la controverse), ont confirmé le rôle social de l'abstract. Ces éléments n'appartiennent plus au monde scientifique mais au domaine de l'humain.

En rapprochant les paramètres pris en considération pour intégrer l'article scientifique dans un genre et les aspects mis en lumière par l'analyse de l'abstract, on constate que ces derniers sont les lieux des variations les plus importantes, voire de disparités. En effet, les aspects liés au profil socio-culturel sont le reflet, l'expression la plus profonde et la moins consciente, de la culture individuelle et collective des auteurs qui, en quelque sorte, se «trahissent ».

21 À travers cette série d'étapes, le linguiste réalise l'importance du non-dit qui se cache derrière les mots et découvre que les modèles anglais ne correspondent pas à la vision qu'ont les Français de la réalité économique, éducative et sociale. Celle-ci pose alors le problème de l'adaptation ou de l'imitation, point qui sera abordé ultérieurement dans la discussion. 
L'étude contrastive a fourni des schémas d'organisation du discours et des informations sur la perception du monde différents. Les modèles établis par la suite ont permis la comparaison. La même démarche a été adoptée pour les introductions pour lesquelles les résultats méritent d'être mentionnés.

\section{Schématisation}

Tableau 0

\begin{tabular}{|l|l|}
\hline Fonctions profession. & $\begin{array}{l}\text { Définition, Référenciation, Présentation, Description, } \\
\text { Description/discussion, Discussion }\end{array}$ \\
\hline $\begin{array}{l}\text { Positionnement dans } \\
\text { le temps }\end{array}$ & $\begin{array}{l}\text { *Temps d'avant la recherche, Temps des activités } \\
\text { scientifiques, Temps de l'écriture. } \\
\text { *Atemporel, passé lointain, passé récent, présent }\end{array}$ \\
\hline $\begin{array}{l}\text { Fonction sociale Relation avec } \\
\text { extérieur }\end{array}$ & \begin{tabular}{l} 
Connu, Nouveau \\
\hline
\end{tabular} \\
\hline
\end{tabular}

Le tableau 0 représente les aspects pris en considération pour la construction des modèles. Les tableaux suivants, ci-dessous, exposent alors les modèles qui se dégagent des analyses. La première ligne y exprime les différentes phases, c'est-à-dire la position dans le texte et le type de fonction scientifique. Les deux lignes suivantes mettent en lumière la chronologie, la perception de l'espace-temps. Enfin, la dernière ligne révèle la nature du message, par conséquent la relation qu'entretient l'auteur avec ses lecteurs et, au-delà, la stratégie mise en œuvre pour promouvoir son travail.

On notera que 3 textes anglais et 5 textes français sont inclassables, les auteurs ayant adopté un traitement marquant fortement leur individualité. Toutefois, il faut comprendre que pour les modèles français 1 et 4 , deux options ont été rapprochées malgré des disparités, le nombre et la nature des variantes étant déjà suffisamment marqués.

Modèles pour l'informatique - 49 abstracts anglais

Tableau 1. Modèle 1 (13 textes)

\begin{tabular}{|l|l|l|}
\hline $\begin{array}{l}\text { 1- Présentation/ } \\
\text { Interprétation }\end{array}$ & 2-Description & 3-Discussion \\
\hline Temps de l'écriture & Temps des activités scientifiques (phase finale) & Temps de l'écriture \\
\hline Présent & Passé récent & Présent \\
\hline Nouveau & Nouveau & Nouveau \\
\hline
\end{tabular}


Tableau 2. Modèle 2 (24 textes)

\begin{tabular}{|l|l|}
\hline 1- Présentation/Interprétation & 2- Discussion \\
\hline Temps de l'écriture & Temps de l'écriture \\
\hline Présent & Présent \\
\hline Nouveau & Nouveau \\
\hline
\end{tabular}

Tableau 3. Modèle 3 (9 textes)

\begin{tabular}{|l|l|}
\hline $\begin{array}{l}\text { 1- Présentation/ } \\
\text { Interprétation }\end{array}$ & 2- Description/Discussion \\
\hline Temps de l'écriture & $\begin{array}{l}\text { Temps des activités scientifiques (phase finale)/Temps de } \\
\text { l'écriture }\end{array}$ \\
\hline Présent & Passé récent/Présent \\
\hline Nouveau & Nouveau \\
\hline
\end{tabular}

\section{Modèles pour l'informatique - 52 abstracts français}

Tableau 4. Modèle 1 (10+3 textes)

\begin{tabular}{|l|l|}
\hline 1- Présentation/Interprétation & 2- Discussion \\
\hline Temps de l'écriture & Temps de l'écriture \\
\hline Présent & Présent \\
\hline Nouveau & Nouveau \\
\hline
\end{tabular}

Tableau 5. Modèle 2 (14 textes)

\begin{tabular}{|l|l|}
\hline 1- Présentation & 2- Description \\
\hline Temps de l'écriture & $\begin{array}{l}\text { Temps des activités scientifiques } \\
\text { (Phase de raisonnement) }\end{array}$ \\
\hline Présent & Chronologie dans le passé \\
\hline \hline Nouveau & Nouveau \\
\hline
\end{tabular}


Tableau 6. Modèle 3 (10 textes)

25

\section{Tableau 7. Modèle 4 (5+5 textes)}

\begin{tabular}{|l|l|l|}
\hline 1- Référenciation & 2- Présentation & 3-Discussion \\
\hline Temps d'avant la recherche & Temps de l'écriture & Temps de l'écriture \\
\hline \hline Passé lointain & Présent & Présent \\
\hline Connu & Nouveau & Nouveau \\
\hline
\end{tabular}

Tableaux récapitulatifs d'organisation : Abstracts et introductions d'informatique

\section{Description}

Temps des activités scientifiques et du raisonnement

Chronologie dans le passé

Nouveau

Le tableau 8 résume les modèles d'organisation précédents et, en informant sur le nombre de textes, permet de comparer les deux corpus. Le deuxième tableau montre les résultats obtenus pour les introductions selon la même approche. On notera la variation au niveau de la description posée comme optionnelle mais qui n'altère guère l'homogénéité des résultats pour le corpus anglais.

Tableau 8. Anglais : 49 abstracts - français : 52 abstracts

\begin{tabular}{|c|c|c|c|c|c|c|}
\hline Position & Présentation & $\begin{array}{l}\text { Présentation } \\
\text { Interprétation }\end{array}$ & Description & $\begin{array}{l}\text { Description/ } \\
\text { Discussion }\end{array}$ & Discussion & $\begin{array}{l}\text { Référen- } \\
\text { ciation }\end{array}$ \\
\hline 1e angl & & 46 & & & & \\
\hline 2e angl & & & 13 & 9 & 24 & \\
\hline 3e angl & & & & & 13 & \\
\hline $1 \mathrm{e} f r$ & 14 & 13 & 10 & & & 5 \\
\hline 2e fr & 5 & & 14 & & 13 & \\
\hline $3 e \mathrm{fr}$ & & & & & 5 & \\
\hline
\end{tabular}

Tableau 9. Anglais : 50 introductions - français : 52 introductions

\begin{tabular}{|l|l|l|l|l|l|}
\hline Position & Définition & Référen-ciation & Présentation & Description/Discussion & Discussion \\
\hline 1e angl & & 44 & & & \\
\hline
\end{tabular}




\begin{tabular}{|l|l|l|l|l|l|}
\hline 2e ang & & & & 44 & \\
\hline \hline e fr & 9 & 22 & 20 & & \\
\hline 2e fr & & 21 & 22 & 8 & \\
\hline 3e fr & & 5 & 9 & 29 & \\
\hline 4e fr & & & & 5 & 9 \\
\hline
\end{tabular}

La comparaison souligne les disparités qui existent entre les productions écrites des deux communautés, leur nature et leurs lieux. L'avantage de l'analyse contrastive réside alors dans le fait qu'elle engendre la prise de conscience sur l'origine de certaines difficultés liées à la rédaction en anglais et le sentiment d'avoir enfin des éléments concrets, posés explicitement, d'ordre tant qualitatif que quantitatif. En effet, les Français font souvent allusion à ces différences dont ils sentent l'existence de manière intuitive sans pouvoir les définir ni trouver de remèdes.

31 Le tableau qui suit schématise la forme la plus conventionnelle de modèle d'abstract et d'introduction en anglais pour l'informatique. Celui-ci constitue alors l'outil sur lequel nous avons fondé notre réflexion qui nous incite à admettre l'idée d'une normalisation par l'acceptation d'un modèle type en anglais auquel les non-anglophones peuvent se conformer plus ou moins selon leur culture. L'adaptabilité dépend, au départ, de la connaissance des phénomènes que nous venons de décrire.

Modèles pour les textes d'informatique anglais

\begin{tabular}{|l|l|l|}
\hline \multicolumn{1}{|c|}{ Abstracts } & \multicolumn{1}{|c|}{ Introductions } & \\
\hline Présentation-Discussion & Référenciation & - Discussion \\
Temps de l'écriture & Tps d'avant la recherche & - Tps de l'écriture \\
Présent & Passé lointain & - Présent \\
Nouveau & Connu & - Nouveau \\
\hline
\end{tabular}

Les analyses effectuées selon la même méthodologie sur les abstracts et les introductions en mécanique ont fourni des résultats sensiblement comparables. Elles font apparaitre à nouveau un modèle anglais fortement marqué en comparaison avec les options françaises, et les divergences sont de même nature bien qu'elles varient par rapport à l'informatique. En revanche, la modélisation des textes de mathématiques s'est avérée impossible, même pour le corpus anglais, tant les individualités des auteurs étaient incompatibles avec la conformisation à des règles normatives.

\section{Validation}

Une première expérimentation a été effectuée afin de valider les modèles anglais. Quatre abstracts français ont été traduits en anglais par des scientifiques de langue française, spécialistes du domaine; seules les erreurs de langue ont été corrigées par nos soins. 
Ensuite, chaque première version a été "détruite " puis « reconstruite » par la même personne et à nouveau corrigée afin de produire une version entièrement reformulée selon le modèle anglais et les résultats d'analyse. Les deux traductions révisées ont été soumises à des «referees" anglophones, américain, canadien et britannique, dont les travaux portent sur les mêmes sujets. Il s'avère que l'application du modèle anglais pour les reformulations entraîne un meilleur accès aux informations et un confort de lecture accru. Les conclusions confirment la nécessité de dissocier les éléments purement linguistiques du contenu des messages. Elles soulignent également l'intérêt d'assurer auprès des anglophones une bonne réception. Certaines versions de départ ont été considérées comme particulièrement mauvaises. Il en résulte que la fabrication d'un nouveau texte à partir du modèle s'avère positive bien que cette première tentative de validation ait porté sur un échantillon insuffisant de textes. Un abstract d'informatique est présenté ci-dessous accompagné des deux versions en anglais afin d'illustrer la méthode.

\begin{abstract}
original français
Identification d'un objet 3D par mise en correspondance entre une seule image et un modèle

Nous présentons ici une méthode d'identification et de localisation d'objets polyédriques à partir d'une image de segments du modèle face-arête-sommet et du graphe d'aspects de l'objet. Elle est fondée sur la recherche d'hypothèses dans un graphe de comptabilité topologique. Les nœuds en sont des correspondances entre chaînes de segments extraites de l'image et faces du modèle. Elles sont établies sur des critères de nombre de côtés. Les arcs reliant les nœuds font appel à des critères de visibilité (graphe d'aspects) et de connexité. Un apport important est fait au niveau du concept de compatibilité topologique globale. par opposition à celui de compatibilité mutuelle. L'expérimentation a montré la validité de cette méthode pour identifier des objets polyédriques convexes.

\section{Traduction}

3D Objects Identification by matching between a single image and a model

This paper describes a method to identify and locate an object from segments image, face-edge-vertex model, and aspect graph of the object. It is based upon a hypothesis search in a topological compatibility graph. Each node is a matching between a segments chain and a face in the object model. The matching criterion is the number of sides. Arcs between nodes correspond to visibility (aspects graph) and connexity criteria. Our contribution is about global topological compatibility concept as opposed to mutual one. Experiments proved the interest of this method to identify convex polyhedral objects from a single view.

\section{Reformulation}

This paper describes a method for identifying and locating a 3D object from segmented images, using a surface-edge-vertex model and the aspect graph of the object. The algorithm is based on computing hypotheses from a topological compatibility graph. Experiments proved the interest of the method for identifying convex polyhedral objects. Our contribution lies in choosing the global topological compatibility concept rather than the mutual compatibility concept.
\end{abstract}

Il serait nécessaire de renouveler cette procédure de validation avec un nombre plus élevé de textes et surtout un seul auteur français afin d'éviter les déviations de nature scientifique, problème qui s'est posé dans notre cas.

Les résultats présentés ne doivent pas être généralisés. Nous ne pouvons pas prétendre en tirer des conclusions hâtives sur l'article scientifique en anglais et en français, des études de plus grande envergure et portant sur d'autres domaines sont alors nécessaires. Cependant, il est permis de reconnaître la possibilité de modéliser des écrits et de relever 
des points positifs pour l'application, lesquels vont être présentés dans la discussion. Nous posons également l'hypothèse que cette méthode de modélisation peut être transférée à d'autres textes et pour d'autres sujets pourvu que l'intervention de l'humain soit marquée, par exemple les rapports de cas en médecine.

\section{Discussion}

\section{Avantages pour l'apprentissage de l'anglais}

La fiabilité de la méthode dépend en premier lieu du principe d'informatisation de l'analyse. Les résultats statistiques ainsi que le concordancier contribuent à fournir un matériau de départ riche, surtout irréfutable, que l'analyse artisanale et l'œil nu ne sont pas toujours en mesure de déceler.

Une approche différente de la lecture de documents authentiques constitue, à nos yeux, un point essentiel qui, de surcroît, semble particulièrement intéressant à développer dans le cadre de l'apprentissage de la langue. En encourageant le repérage, en le guidant pour l'orienter vers des points clés, on éveille la prise de conscience de phénomènes implicites et on provoque un autre regard sur la communication scientifique écrite.

Du point de vue linguistique, il est souhaitable de dépasser le stade du visible et de susciter des mécanismes nouveaux. Cette méthode contraint d'abord à dissocier la qualité de la langue et celle du message, comme la validation appliquée à l'abstract d'informatique présenté l'a confirmé, puis à établir la relation entre la langue et les intentions de communication tout en intégrant les conventions en usage. Une application effectuée à partir de l'abstract suivant permet de mieux percevoir les deux points mentionnés.

Recent Progress in Real-Time Image Analysis for Real World Traffic Analysis.

There has been a great demand for more innovative and effective traffic monitoring and control system to tackle the continuing rise of traffic levels. This paper reviews the computer vision application of road traffic monitoring, and briefly highlights major achievements whilst identifying difficulties and discussing their suitable solutions. Some of the authors' relevant trials on traffic image segmentation and object re-identification are presented.

En partant de sa propre langue et de son expérience en tant que Français, on peut mettre l'accent sur les relations français/anglais, science et culture; grâce à l'approche contrastive, on invite ainsi les auteurs à repérer des points de vue universels et spécifiques en s'interrogeant tout d'abord sur les mécanismes de leur propre langue et leur mode de communication. Cette prise de conscience de la possibilité d'exprimer différemment des notions universelles, l'enchânement logique des idées ou le temps, selon l'origine du locuteur, semble offrir le meilleur point de départ vers la démarche qui conduira à imiter le modèle. Enfin, le fait de reconnaître l'existence de ces modèles et conventions conduit à confirmer la tendance à la normalisation et l'importance du sentiment collectif des scientifiques anglo-saxons, bien qu'il soit plus juste de préciser « anglo-américain ». Ce paramètre ne nous semble pas totalement dénué d'intérêt.

Enfin, la faculté d'adaptation des scientifiques français au modèle anglais sera d'autant plus accrue que le formateur sera en mesure de les aider à développer leurs propres systèmes d'investigation, à savoir leur indiquer comment et où diriger leur regard. Sa contribution en ce qui concerne l'acceptation intellectuelle, puis affective, et l'adaptation 
à des règles de fonctionnement différentes peut surprendre dans un environnement scientifique, mais nous croyons qu'elle fait partie intégrante de l'apprentissage à la rédaction scientifique. De ce point de vue, il ne s'agit donc pas de donner des recettes prêtes à l'emploi qui ne seraient pas transférables, mais de développer un savoir-faire et de provoquer de nouveaux mécanismes afin de faire face à toute situation. L'épreuve de rédaction devient ainsi plus active que la simple traduction du français vers l'anglais. Quelques expériences ont été menées auprès de nos étudiants et de nos candidats au module de rédaction scientifique dans le cadre du Pôle universitaire européen à partir de tâches conçues spécifiquement. Les réticences et réactions hostiles de certaines personnes ont laissé rapidement la place à la bonne volonté et à la mise en œuvre. Cependant, dans l'état actuel de notre recherche, il est utile d'en rappeler les limites.

\section{Limites}

Le premier point devant être mentionné se rapporte aux conditions d'applicabilité de la modélisation. Il est risqué d'envisager l'existence de modèles en anglais sans avoir analysé au préalable un nombre suffisant de textes. En outre, toutes les sections ne se prêtent pas à ce genre d'analyse. Il faut également tenir compte des cultures inhérentes à certaines disciplines, institutions ou aux contextes de publication, ce qui est le cas pour une partie des mathématiques. Enfin, il faut admettre la possibilité de comportements atypiques des auteurs qui ne se soumettent pas à une forme de normalisation collective.

Le point qui incite à la plus grande prudence porte sur le côté réducteur de la méthode. Il est vrai que la conformité à un modèle unique tend à exclure les variations, les initiatives personnelles, et à long terme à assujettir la langue à la fonction en niant la personne qui émet le message. C'est ainsi que l'enseignant pourrait, dans le cadre d'une application sans le moindre discernement, proposer un travail se restreignant à une partie de la langue, à n'accepter de la part des apprenants que des écrits conformes et à pénaliser toute forme d'écart. Par conséquent, la vigilance s'impose pour ne pas dépasser les conditions d'utilisation, ne pas dévier, c'est-à-dire appliquer les modèles à toute forme d'écrit ni emprisonner les apprenants dans une méthode perçue comme un carcan; ici, nous avons envisagé des conditions précises et un échantillon limité.

En revanche, l'approche offre une sorte de noyau central, ou tronc commun, avec des constantes applicables à diverses formes de discours. Que le raisonnement et l'agencement des idées soient formulés dans des documents scientifiques ou dans des résumés traitant de culture générale, il est prudent de garder à l'esprit les informations positives apportées par la conception des modèles, par exemple sur la cohérence thématique ou l'ordonnancement des idées.

Enfin, nous tenons à préciser que la langue n'est pas figée et que la science est en perpétuelle mouvance. Par conséquent, nous ne pouvons prétendre poser les modèles de façon statique. L'évolution de la langue, des règles en usage, des phénomènes de mode qui interviennent également dans le monde scientifique incite à refuser la rigidité et à accepter la valeur des analyses à un moment donné. Le formateur semble donc contraint de suivre cette évolution.

En conclusion, il est indispensable de situer le débat en délimitant le champ et les conditions d'application de notre méthode afin d'éviter les écueils. L'intérêt d'une telle démarche consiste, pour les Français qui rédigent de l'anglais scientifique, à déclencher un processus de lecture, de recherche de messages et de création de leur propre matériau. 
Elle tente de répondre à des besoins sans nier la personnalité des utilisateurs qui deviennent réellement actifs au moment de l'expression de leurs idées. C'est donc une approche également différente de la rédaction qui est souhaitable. L'objectif essentiel, à nos yeux, est de provoquer une prise de conscience de faits plus ou moins cachés ainsi que des interrogations dans le cadre de ce qu'on pourrait appeler un transfert de culture, plutôt que la traduction du français vers l'anglais, pratique qui demeure encore très fréquente dans la communauté scientifique française.

\section{BIBLIOGRAPHIE}

Ayers, G. 1993. « A preliminary investigation of abstracts through a genre-analysis of the short texts accompanying articles and letters in the scientific journal Nature ». M.A. Dissertation, Birmingham : University of Birmingham.

Banks, D. 1994. Writ in Waters: Aspects of the Scientific Journal Article. Brest : ERLA, Université de Bretagne Occidentale.

Bazerman, C. 1988. Shaping Written Knowledge. The Genre and Activity of the Experimental Article in Science. Madison : University of Wisconsin Press.

Beacco, J-C. \& D. Lehmann (dir.). 1990. « Publics spécifiques et communication spécialisée ». Le français dans le monde, Collection Recherches et applications, Paris : Hachette, 142-149.,

Bhatia, V. K. 1995. « Applied genre analysis and ESP ». The Journal of TESOL France 1/1, Functional Approaches to Written Text: Classroom Applications, 161-180.

Cooke, R. 1993. « Learning to publish in English: How can French researchers bridge the gap? ». ASp 1, 463-473.

Crosnier, E. 1995. « Les scientifiques français et la publication en langue anglaise : contraintes, obstacles et perspectives de solutions d'aide à la rédaction. Étude contrastive de corpus anglaisfrançais informatisés d'informatique, de mathématiques et de mécanique (Titres, abstracts et introductions). Thèse de doctorat préparée sous la direction de J.-P. Soula, Toulouse : Université de Toulouse le Mirail.

Defaye, J. R. 1985. « Repérage et analyse des opérations discursives dans quelques textes d'anglais de spécialité (géologie sédimentaire) ». Cahiers de l'ILSER 4, 29-53.

Dudley-Evans, T. 1995. « Genre models for the teaching of academic writing to second language speakers: Advantages and disadvantages ». The Journal of TESOL France 1/1, Functional Approaches to Written Text: Classroom Applications, 181-192.

Hopkins, A. \& T. Dudley-Evans. 1988. «A genre-based investigation of the discussion sections in articles and dissertations ». English for Specific Purposes 7/2, 113-121.

Johns, A. M. 1994. « LSP and culture: A special relationship ». ASp 5-6, 11-19.

Miller, T. 1993. " The introduction to the research article from a discourse perspective ». ASp 2, $1-26$.

Péry-Woodley, M-P. 1993. Les écrits dans l'apprentissage. Paris : Hachette FLE, série Références. 
Salager-Meyer, F. 1990. « Discoursal flaws in medical English abstracts: A genre analysis per research and text type ». Text 10, 365-384.

Salager-Meyer, F. 1992. «A text-type and move analysis study of verb tense and modality distribution in medical English abstracts ». English for Specific Purposes 11/2, 93-113.

Swales, J. 1981. « Aspects of article introductions ». Aston ESP Research Report 1, Birmingham : Language Studies Unit, University of Aston.

\section{RÉSUMÉS}

La modélisation se définit comme l'opération qui consiste à construire le schéma d'un processus réel que l'on veut élucider. Dans notre cas, il s'agit de la communication scientifique écrite. Notre recherche porte sur une analyse contrastive d'extraits d'articles français et anglais. Nous exposons la méthodologie, à savoir le repérage d'indices, leur interprétation selon une approche fonctionnelle et notionnelle et enfin la constitution de modèles fondée sur le concept de récurrence. Nous montrons ensuite une première expérimentation qui tend à valider les résultats. L'observation de quelques-unes des difficultés rencontrées par les scientifiques de langue française nous conduit à envisager la modélisation comme aide à la rédaction scientifique tout en gardant à l'esprit ses limites. Nous posons enfin l'hypothèse que l'application à d'autres types de discours et d'autres disciplines est possible.

Modelling consists in constructing the pattern of a real process which is to be clarified, notably written scientific communication. Our investigation concerns a contrastive analysis of specific sections in French and English papers. The main steps of the methodology are exposed, i.e., retrieving clues, giving functional and notional interpretations, and building models based on recurrence. Our first experiment aims to validate the results. After determining some of the obstacles encountered by French scientists when writing scientific English, we will consider modelling to be of any help while mentioning its limitations. This approach can presumably be applied also to other types of discourse and disciplines.

\section{INDEX}

Mots-clés : analyse contrastive, modélisation, rédaction d'article scientifique, schéma

Keywords : contrastive analysis, modelling, pattern, scientific paper writing

\section{AUTEUR}

\section{ELIZABETH CROSNIER}

Elizabeth Crosnier enseigne l'anglais à l'INSA de Toulouse. Elle intervient dans le module de rédaction scientifique pour le Pôle universitaire européen à Toulouse. Elle travaille également à la traduction et à la relecture d'articles avec des chercheurs des départements de GEII et G2M de l'INSA. Elle effectue ses recherches sur les problèmes et les besoins des scientifiques français en matière de publication spécialisée en langue anglaise. Elle mène une réflexion sur les applications pédagogiques de l'anglais de spécialité en milieu francophone. Avec les autres membres de l'équipe de recherche du CERDIC, elle travaille sur la présentation scientifique orale en vue de la fabrication d'un CD-ROM comme outil d'aide pour les Français. elizabeth.crosnier@orange.fr 